\title{
Gender Differences in Rapid Eye Movement-Related Sleep Disordered Breathing
}

\author{
Minjung Youn'1, Joo Young Kwon1, Kyu Sun Lee ${ }^{1,2}$, Jung Hyun Park¹, Hyang Woon Lee ${ }^{1,2^{*}}$ \\ ${ }^{1}$ Department of Neurology, Ewha Womans University School of Medicine and Ewha Medical Research Institute, \\ Seoul, Korea \\ ${ }^{2}$ Department of Neurology, Ajou University School of Medicine, Suwon, Korea \\ Email: ${ }^{*}$ leeh@ewha.ac.kr
}

Received 18 December 2014; accepted 1 January 2015; published 19 January 2015

Copyright (C) 2015 by authors and Scientific Research Publishing Inc.

This work is licensed under the Creative Commons Attribution International License (CC BY).

http://creativecommons.org/licenses/by/4.0/

(c) (i) Open Access

\section{Abstract}

Sleep disordered breathing (SDB) is an independent risk factor for cardiovascular disease. It is known to be associated more frequently with men than women, particularly in the premenopausal age range. The goal of this study is to evaluate gender differences among Korean patients diagnosed with SBD. This study included 309 patients who visited our Sleep Clinic due to sleep-related symptoms and were diagnosed with SDB by overnight polysomnography (PSG). We analyzed age, gender, body mass index, various PSG indices including sleep stages, apnea-hypopnea index (AHI) and AHI ratio in rapid eye movement (REM) versus non-REM (NREM) sleep stages (R:N ratio). Of those 309 patients diagnosed with obstructive sleep apnea, $217(70.2 \%)$ were men (mean age $51.05 \pm 12.64$ years) and $92(29.8 \%)$ were women (mean age $64.53 \pm 10.43$ years). The mean AHI during total sleep time was $30.34 \pm 21.17$ in men and $21.47 \pm 17.14$ in women $(P<0.001)$. The AHI in NREM sleep was higher in men than in women $(30.97 \pm 22.39$ versus $20.19 \pm 18.17, P<0.001)$, whereas the AHI in REM sleep was not significantly different between men and women $(25.73 \pm$ 21.61 versus $28.00 \pm 21.76, P=0.402$ ). REM SDB with $R: N$ ratio higher than 2.0 was more frequently observed in women than in men, $34.8 \%(32 / 92)$ of women, compared with $11.9 \%$ $(26 / 217)$ in men $(P<0.001)$. Interestingly, the mean $R: N$ ratio tended to decrease with increasing age in women, but remained relatively constant in men. The prevalence of REM SDB was higher in women than in men, and the gender difference was the most prominent in women with the age < 60 years old compared to age-matched and older men or women $\geq 60$ years old. These findings suggest the possibility of different pathophysiologic mechanisms of SDB between genders and also between NREM versus REM sleep, which can be partly explained by the influence of female sex hormones.

\footnotetext{
"Corresponding author.
} 


\title{
Keywords
}

\author{
Women, Sleep Disordered Breathing, Obstructive Sleep Apnea, Rapid Eye Movement Sleep, \\ Apnea-Hypopnea Index
}

\section{Introduction}

Sleep disordered breathing (SDB) is an independent risk factor for cardiovascular disease. It is well known from previous epidemiological studies that men have higher prevalence of SDB or obstructive sleep apnea (OSA) than women, although the incidence in women increased dramatically in postmenopausal age [1] [2].

Rapid eye movement (REM) sleep-related SDB is a phenomenon that sleep apnea and hypopnea appear predominately during the period of REM sleep, and defined as that the ratio of apnea-hypopnea index (AHI) between REM and non-REM (NREM) sleep (R:N ratio) is higher than 2.0 [3]-[6]. REM SDB has been estimated to be in wide range, between $7 \%$ and $62 \%$ of all OSA patients [1]-[5]. Interestingly, these studies have suggested that the REM SDB has a unique prevalence in different age and gender groups, which might be influenced by obesity and/or anatomical differences of upper airway between genders or during REM sleep.

Previous literature suggest that upper airway muscle tension could be decreased most during REM sleep, and airway resistance increased, and thus could induce airway collapse [6] [7]. Airway tension during REM sleep could be the lowest as compared to other sleep stages since it is associated with a decrease of chemical receptor responses to hypoxia and hypercapnia in the medulla oblongata. OSA patients could have more SDB related to low oxygen saturation during REM sleep [8] [9], therefore extending our knowledge related to REM SDB has a clinical significance. However, data related to the difference in REM SDB between gender and age groups are still lacking in Asia, especially in Korea. This study aimed to compare sleep indices related SDB and to analyze various clinical factors, especially age and gender in Korean patients who were diagnosed with OSA based on overnight polysomnography (PSG). Our study revealed a comparable finding to the previous epidemiological studies in North American and European countries which showed women, especially during the premenopausal age, having higher percentage in REM SDB [1]-[5].

\section{Methods}

\subsection{Subjects}

This study recruited 309 patients diagnosed with SDB among those 1030 subjects who had visited Sleep Clinic due to various sleep related symptoms and performed overnight PSG at Ewha Womans University Medical Center between July 1, 2005 and July 1, 2014.

Inclusion criteria were: 1) complete clinical and PSG records; 2) confirmed diagnosis of OSA based on clinical and PSG findings; and 3) age between 30 - 79 years. Exclusion criteria were: 1) age less than 29 or older than 80 years old; 2) other cardiopulmonary disorders such as chronic heart failure or chronic obstructive pulmonary diseases; and 3) any other neurological or psychological disorders, such as neurodegenerative diseases, major depression or schizophrenia. However, women with oral contraceptives, hormonal replacement therapy, or history of total abdominal hysterectomy with salpingo-oophorectomy were not excluded in the current study.

\subsection{Sleep Questionnaires and Night Polysomnogram (PSG)}

Subjects completed a self-administered sleep questionnaire, the Pittsburgh Sleep Quality Index (PSQI) [10], the Stanford Sleepiness Scale (SSS) [11], the Epworth Sleepiness Scale (ESS) [12], and the Beck Depression Inventory (BDI) [13] based on experiences over the previous month.

We performed one night in-lab attended PSG (Comet XL Lab-based PSG, TWin PSG software, Grass-Telefactor, RI, USA). All nighttime sleep recordings started after lights off according to the usual bedtime of subjects and ended at their wake-up time the next morning. Cardiorespiratoroy monitoring was applied including bilateral monopolar central and occipital EEG with 4 channels (C3-A2, C4-A1, O1-A2, O2-A1), electro-oculogram (left outer canthus-A1, right outer canthus-A2), chin and bilateral tibialis anterior surface electromyography (EMG), thermistor, pressure transducer air flow (PTAF), snoring sound registration by microphone, thoracic 
and abdominal belts to measure respiratory movements (respiratory inductance plethysmography), electrocardiogram (modified V2 lead), finger pulse oximetry, and body position sensor.

Sleep staging and detection of respiratory events were based on Rechtschaffen and Kales Method [14]. Sleep apnea was defined as absence of respiration longer than 10 seconds, and hypopnea was defined as obvious reduction of respiration associated with EEG arousal or decreased oxygen saturation more than 3\%. OSA was diagnosed when the apnea-hypopnea index (AHI) was higher than 5 per hour. REM SDB was determined by calculating the REM AHI to NREM AHI ratio (R:N) and defined as R:N $\geq 2$ and NREM AHI $\leq 15$.

We analyzed sleep stages, gender, body mass index, and other PSG indices including apnea-hypopnea index (AHI) and AHI ratio in REM versus NREM sleep stages (R:N ratio).

\subsection{Statistical Analysis}

Student t-test was used to compare clinical and sleep variables between men and women, between REM and NREM sleeps. Chi-square test was used to compare the prevalence of REM SDB and NERM SDB between men and women. All the statistical analyses were performed using the SPSS version 11.0 (SPSS Inc., Chicago, IL, USA). The level of significance was set at 0.05 allowing for $5 \%$ of alpha error.

\section{Results}

\subsection{Clinical Sleep Characteristics of Patients Compared by Gender}

The clinical and sleep characteristics of all patients are summarized in Table 1. Of those 309 patients diagnosed with OSA, 217 (70.2\%) were men and 92 (29.8\%) were women. The mean age was older in women than in men $(P<0.001)$, but body mass index (BMI) was not different between men and women, nor between patients with REM SDB and NREM SDB. The mean AHI during total sleep time were $30.34 \pm 21.17$ in men and $21.47 \pm$ 17.14 in women $(P<0.001)$. The AHI in NREM sleep was higher in men than in women $(30.97 \pm 22.39$ versus $20.19 \pm 18.17, P<0.001$ ), whereas the AHI in REM sleep was not significantly different between men and women ( $25.73 \pm 21.61$ versus $28.00 \pm 21.76, P=0.402)$. The mean $\mathrm{R}: \mathrm{N}$ ratio was significantly higher in women than men $(P<0.001)$. In addition, REM SDB with R:N ratio higher than 2.0 was more frequently observed in $34.8 \%$ (32/92) of women, compared with 11.9\% (26/217) in men $(P<0.001)$ (Figure 1).

\subsection{Polysomnographic Findings of Patients}

Respiratory events recorded from PSG according to age and gender were summarized in Table 2. Although the AHI was higher in men than in women both age groups $<60$ and $\geq 60$ years old $(P=0.003$ and $P=0.020$, respectively), the differences of mean value of AHI between men and women dramatically decreased from 12.53 to 7.89 with increasing age. Interestingly, the mean R:N ratio tended to decrease with increasing age in women, but remained relatively constant in men (Figure 2).

Table 1. Clinical and sleep characteristics of patients according to gender.

\begin{tabular}{cccccccc}
\hline & $\begin{array}{c}\text { Total } \\
(\mathrm{N}=309)\end{array}$ & $\begin{array}{c}\text { Men } \\
(\mathrm{n}=217)\end{array}$ & $\begin{array}{c}\text { Women } \\
(\mathrm{n}=92)\end{array}$ & $P$-value & $\begin{array}{c}\text { REM SDB } \\
(\mathrm{n}=58)\end{array}$ & $\begin{array}{c}\text { NREM SDB } \\
(\mathrm{n}=251)\end{array}$ & $P$-value \\
\hline Age (years) & $55.06 \pm 13.50$ & $51.05 \pm 12.64$ & $64.53 \pm 10.43$ & $<0.001^{* *}$ & $53.12 \pm 13.60$ & $54.11 \pm 14.52$ & 0.915 \\
BMI $\left(\mathrm{kg} / \mathrm{m}^{2}\right)$ & $25.56 \pm 3.81$ & $25.70 \pm 3.46$ & $24.19 \pm 4.15$ & 0.170 & $24.91 \pm 4.41$ & $25.76 \pm 3.58$ & 0.077 \\
ESS & $8.53 \pm 7.28$ & $9.49 \pm 7.65$ & $6.10 \pm 5.55$ & $<0.001^{* *}$ & $7.30 \pm 5.64$ & $8.60 \pm 7.60$ & 0.208 \\
AHI & $27.70 \pm 20.43$ & $30.34 \pm 21.17$ & $21.47 \pm 17.14$ & $<0.001^{* *}$ & $21.28 \pm 19.71$ & $26.75 \pm 21.31$ & $0.008^{* *}$ \\
REM AHI & $26.40 \pm 21.65$ & $25.73 \pm 21.61$ & $28.00 \pm 21.76$ & 0.402 & $25.80 \pm 21.36$ & $22.15 \pm 22.93$ & $0.032^{*}$ \\
NREM AHI & $27.76 \pm 21.76$ & $30.97 \pm 22.39$ & $20.19 \pm 18.17$ & $<0.001^{* *}$ & $7.54 \pm 3.10$ & $31.74 \pm 21.39$ & $<0.001^{* *}$ \\
R:N ratio & $1.82 \pm 1.81$ & $1.21 \pm 1.59$ & $2.11 \pm 2.14$ & $<0.001^{* *}$ & $4.77 \pm 2.19$ & $0.83 \pm 0.71$ & $<0.001^{* *}$ \\
\hline
\end{tabular}

Abbreviation: NREM SDB, non rapid eye movement sleep disordered breathing; REM SDB, rapid eye movement sleep disordered breathing; BMI, body mass index; AHI, apnea-hypopnea index; R:N ratio, REM AHI:NREM AHI ratio, ${ }^{*} P<0.05,{ }^{* *} P<0.01$. 
Table 2. Polysomnographic sleep indices of patients according to age and gender.

\begin{tabular}{|c|c|c|c|c|c|c|c|c|}
\hline & \multicolumn{4}{|c|}{ Age $<60$ years } & \multicolumn{4}{|c|}{ Age $\geq 60$ years } \\
\hline & Men & Women & Difference & $P$-value & Men & Women & Difference & $P$-value \\
\hline AHI (total) & $30.08 \pm 21.48$ & $17.70 \pm 12.37$ & 12.53 & $0.003^{* *}$ & $31.53 \pm 19.93$ & $23.25 \pm 18.56$ & 7.89 & $0.020^{*}$ \\
\hline REM AHI & $25.43 \pm 21.37$ & $25.59 \pm 19.38$ & 0.16 & 0.850 & $27.02 \pm 22.63$ & $29.52 \pm 22.65$ & 2.49 & 0.550 \\
\hline NREM AHI & $30.76 \pm 23.01$ & $16.08 \pm 13.23$ & 14.53 & $<0.001^{* *}$ & $31.62 \pm 21.14$ & $22.09 \pm 20.09$ & 9.53 & $0.008^{* * *}$ \\
\hline $\mathrm{R}: \mathrm{N}$ ratio & $1.26 \pm 1.68$ & $2.61 \pm 1.88$ & 1.03 & $0.012^{*}$ & $1.07 \pm 1.26$ & $1.92 \pm 2.27$ & 0.91 & $0.003^{* * *}$ \\
\hline
\end{tabular}

Abbreviation: AHI, apnea-hypopnea index; REM, rapid eye movement sleep; NREM, non rapid eye movement sleep, R:N ratio, REM AHI : NREM AHI ratio, ${ }^{*} P<0.05,{ }^{* *} P<0.01$.

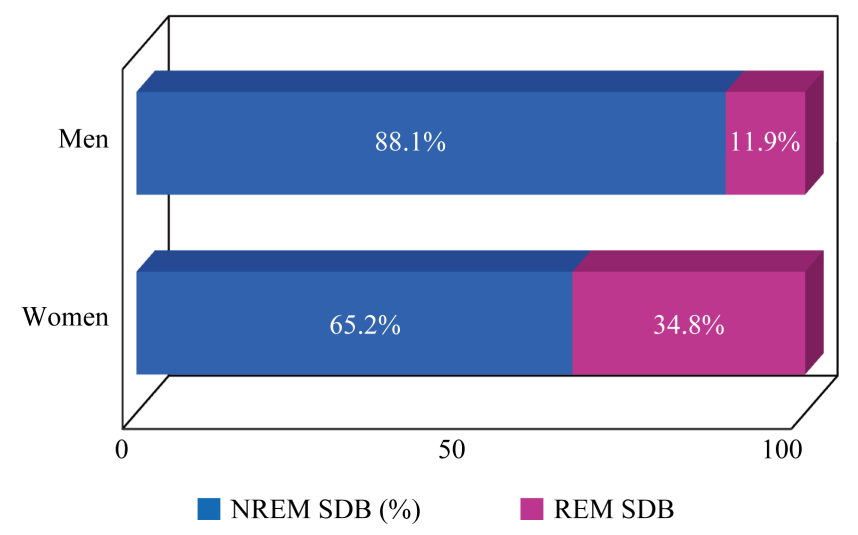

Figure 1. Prevalence of REM-SDB between men and women with SDB.

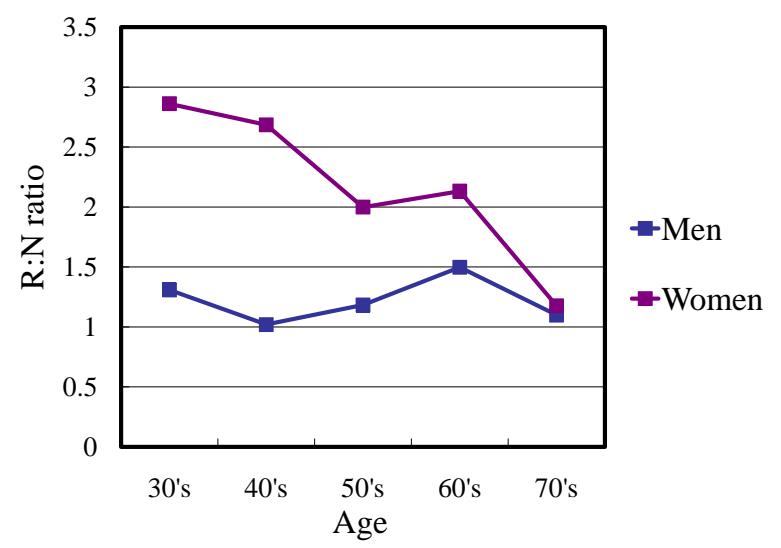

Figure 2. The mean AHI ratio in REM versus NREM sleep stages (R:N ratio) by age in men and women with SDB.

\section{Discussion}

This study was performed to investigate the age and gender differences in Korean patients with REM SDB. Overall, the prevalence of REM-related OSA was higher in women than in men, and the gender difference was the most prominent in women before the age of 60 years, compared with age-matched and older men or women older than 60 years. Interestingly, mean R: $\mathrm{N}$ ratio tended to decrease with increasing age in women, but remained relatively constant in men.

In our study, 32 patients (34.8\%) from 92 women diagnosed with OSA had REM SDB while 26 patients (11.9\%) from 217 men had REM SDB, which showed lower prevalence than a previous study that showed 62\% 
for women and $24 \%$ for men [1], but slightly higher than the study result from another study that reported $24.5 \%$ for women and 7.9\% for men [4]. Most other studies reported with the range of 10\% - 36\% of REM SDB, which was comparable with our results [2] [3] [5]. We assume that such difference of prevalence could be affected by the demographic differences of previous studies. Also, women showed a tendency of R:N ratio decrease as they get older but men maintained an even level without any big difference in our study. In addition to the fact that women had more frequent REM SDB than men unlike the prevalence of overall OSA, the difference in women with different ages suggest possibility of influences from sex hormones, BMI or other factors in REM SDB.

Regarding female hormones, estrogen and progesterone have been shown to increase the activity of the genioglossus muscle, the upper airway dilator [15], with the highest peak phasic and tonic activity in the luteal phase when progesterone effect is dominant. Another study revealed that upper airway resistance was also the lowest in the luteal compared with the follicular phase [16]. Moreover, progesterone has been reported to increase ventilatory reaction to hypoxia and hypercapnia [17] [18], and could induce a protective action regarding SDB and could thus lead to the low overall percentage of women with SDB. But, overall muscle relaxation occurs in REM and thus female hormones' effects on upper airway muscle may decrease. Additionally, the upper airway in women could collapse easily than men due to the smaller relative size of the female upper airway, and its anatomical structure [19]. These factors may make women more vulnerable to SDB during REM. As explained before, a decrease in the chemical receptor responses of medulla oblongata to hypoxia and hypercapnia occurs in REM along with a decrease in the effect of progesterone, could work together to produce a protective effect for women during SDB. Therefore, a gender difference in REM SDB (women having higher prevalence than men) occurred and also the decreased prevalence along with the increase of age in women was believed to occur because of a relative decrease of $\mathrm{R}: \mathrm{N}$ ratio in older postmenopausal women who could not benefit from the protective effect of female hormones against SDB, during all the cycles of sleep, as do women before menopause, and thus their susceptibility to SDB might increase not only during REM but in NREM sleep as well. Conversely, we could apply the reason to men's result that a relatively even R:N ratio was maintained for them without beneficial effects of female sex hormones throughout the various age groups.

Obesity is a well-known risk factor for OSA [20]. During the process of upper airway collapse in NREM, female hormones increase the tension of the upper airway and thus can induce protective effects but, such effects would be less than optimal during REM. As a result, the level of obesity required to induce SDB should be greater in NREM than REM and we can expect women with NREM SDB to have a higher BMI than those with REM SBD. In this study, however, BMI between patients with REM SDB and NREM SDB were not different, nor between men and women.

This study has several limitations. First, the subjects were recruited in the single hospital. Second, female hormones were not measured during the research and there was no investigation into the menstrual cycles or use of hormone therapy and related medications that could affect female hormone secretion. Hormonal replacement therapy, oral contraceptives, history of total abdominal hysterectomy with salpingo-oophorectomy, or hormonal replacement therapy in pre- and postmenopausal women might have some influences on gender differences in SDB. Despite of such limitations, we observed a relatively higher percentage of women with REM SDB compared with men, especially in younger age group $<60$ years. Therefore, this study is meaningful and these results raise awareness for the importance of customized treatments for individual patients with OSA especially in different gender and age groups, which needs more attention for every day clinical practice in sleep clinics.

\section{Conclusion}

In conclusion, the prevalence of REM RDB was higher in women than in men in Korea, and the gender difference was the most prominent in women with younger age before 60 years old, compared with age-matched and older men or women older than 60 years old. These findings suggest different pathophysiologic mechanisms of SDB between gender and also between REM and NREM sleep, which can be partly explained by the influence of female sex hormones.

\section{Acknowledgements}

This research was supported by grants of the Korea Health Technology R\&D Project through the Korea Health Industry Development Institute (KHIDI), funded by the Ministry of Health \& Welfare [H114C1989], and of the Basic Science Research Program through the National Research Foundation (NRF) by the Ministry of Science, 
ICT and future planning, Republic of Korea [2014-R1A2A1A11052103].

\section{References}

[1] O’Connor, C., Thornley, K. and Hanly, P. (2000) Gender Differences in the Polysomnographic Features of Obstructive Sleep Apnea. American Journal of Respiratory and Critical Care Medicine, 161, 1465-1472. http://dx.doi.org/10.1164/ajrccm.161.5.9904121

[2] Resta, O., Carpagnano, G.E., Lacedonia, D., Di Gioia, G., Giliberti, T., Stefano, A. and Bonfitto, P. (2005) Gender Difference in Sleep Profile of Severely Obese Patients with Obstructive Sleep Apnea. Respiratory Medicine, 99, 91-96. http://dx.doi.org/10.1016/j.rmed.2004.05.014

[3] Haba-Rubio, J., Janssens, J.P., Rochat, T. and Sforza, E. (2005) Rapid Eye Movement Related Disordered Breathing: Clinical and Polysomnographic Features. Chest, 128, 3350-3357. http://dx.doi.org/10.1378/chest.128.5.3350

[4] Koo, B., Dostal, J., Ioachimescu, O. and Budur, K. (2008) The Effects of Gender and Age on REM-Related SleepDisordered Breathing. Sleep and Breathing, 12, 257-264. http://dx.doi.org/10.1007/s11325-007-0161-7

[5] Koo, B.B., Patel, S.R., Strohl, K. and Hoffstein, V. (2008) REM-Related Sleep Disordered Breathing: Influence of Age and Gender. Chest, 134, 1156-1161. http://dx.doi.org/10.1378/chest.08-1311

[6] Katz, E. and White, D. (2004) Genioglossus Activity during Sleep in Normal Control Subjects and Children with Obstructive Sleep Apnea. American Journal of Respiratory and Critical Care Medicine, 170, 553-560. http://dx.doi.org/10.1164/rccm.200403-262OC

[7] White, D., Douglas, N., Pickett, C., Weil, J. and Zwillich, C. (1982) Hypoxic Ventilatory Response Decreases during Sleep in Normal Men. The American Review of Respiratory Disease, 125, 286-289.

[8] Douglas, N., White, D., Weil, J., Pickett, C. and Zwillich, C. (1982) Hypercapnic Ventilatory Response in Sleeping Adults. The American Review of Respiratory Disease, 126, 758-762.

[9] Series, F., Cormier, Y. and La Forge, J. (1990) Influence of Apnea Type and Sleep Stage on Nocturnal Postapneic desaturation. The American Review of Respiratory Disease, 141, 1522-1526. http://dx.doi.org/10.1164/ajrccm/141.6.1522

[10] Buysse, D.J., Reynolds 3rd, C.F., Monk, T.H., Berman, S.R. and Kupfer, D.J. (1989) The Pittsburgh Sleep Quality Index: A New Instrument for Psychiatric Practice and Research. Psychiatry Research, 28, 193-213. http://dx.doi.org/10.1016/0165-1781(89)90047-4

[11] Hoddes, E., Zarcone, V., Smythe, H., Phillips, R. and Dement, W.C. (1973) Quantification of Sleepiness: A New Approach. Psychophysiology, 10, 431-436. http://dx.doi.org/10.1111/j.1469-8986.1973.tb00801.x

[12] Johns, M.W. (1991) A New Method for Measuring Daytime Sleepiness: The Epworth Sleepiness Scale. Sleep, 14, 540545.

[13] Beck, A.T., Ward, C.H., Mendelson, M., Mock, J. and Erbaugh, J. (1961) An Inventory for Measuring Depression. Archives of General Psychiatry, 4, 561-571. http://dx.doi.org/10.1001/archpsyc.1961.01710120031004

[14] Rechtschaffen, A. and Kales, A.D. (1968) A Manual of Standardized Terminology, Techniques and Scoring System for Sleep Stages of Human Subjects. Brain Information Service/Brain Research Institute, Los Angeles.

[15] Popovic, R. and White, D. (1998) Upper Airway Muscle Activity in Normal Women: Influence of Hormonal Status. Journal of Applied Physiology, 84, 1055-1062.

[16] Driver, H.S., McLean, H., Kumar, D.V., Farr, N., Day, A.G. and Fitzpatrick, M.F. (2005) The Influence of the Menstrual Cycle on Upper Airway Resistance and Breathing during Sleep. Sleep, 28, 449-456.

[17] Zwillich, C., Natalino, M., Sutton, F. and Weil, J. (1978) Effect of Progesterone on Chemosensitivity in Normal Man. Journal of Laboratory and Clinical Medicine, 92, 262-269.

[18] Shahar, E., Redline, S., Young, T., Boland, L.L., Baldwin, C.M., Nieto, F.J., et al. (2003) Hormone Replacement Therapy and Sleep-Disordered Breathing. American Journal of Respiratory and Critical Care Medicine, 167, 11861192. http://dx.doi.org/10.1164/rccm.200210-12380C

[19] Brooks, L.J. and Strohl, K.P. (1992) Size and Mechanical Properties of the Pharynx in Healthy Men and Women. American Review of Respiratory Disease, 146, 1394-1397. http://dx.doi.org/10.1164/ajrccm/146.6.1394

[20] Ye, L., Pien, G.W. and Weaver, T.E. (2009) Gender Differences in the Clinical Manifestation of Obstructive Sleep Apnea. Sleep Medicine, 10, 1075-1084. http://dx.doi.org/10.1016/j.sleep.2009.02.006 
Scientific Research Publishing (SCIRP) is one of the largest Open Access journal publishers. It is currently publishing more than 200 open access, online, peer-reviewed journals covering a wide range of academic disciplines. SCIRP serves the worldwide academic communities and contributes to the progress and application of science with its publication.

Other selected journals from SCIRP are listed as below. Submit your manuscript to us via either submit@scirp.org or Online Submission Portal.
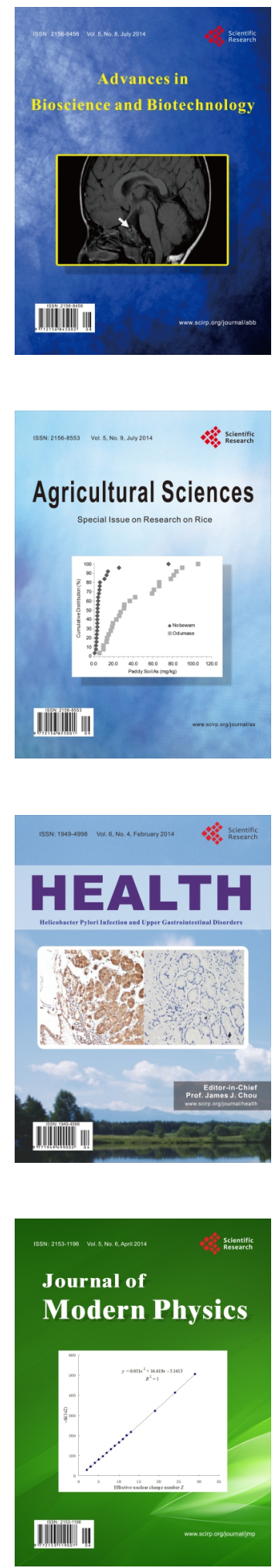
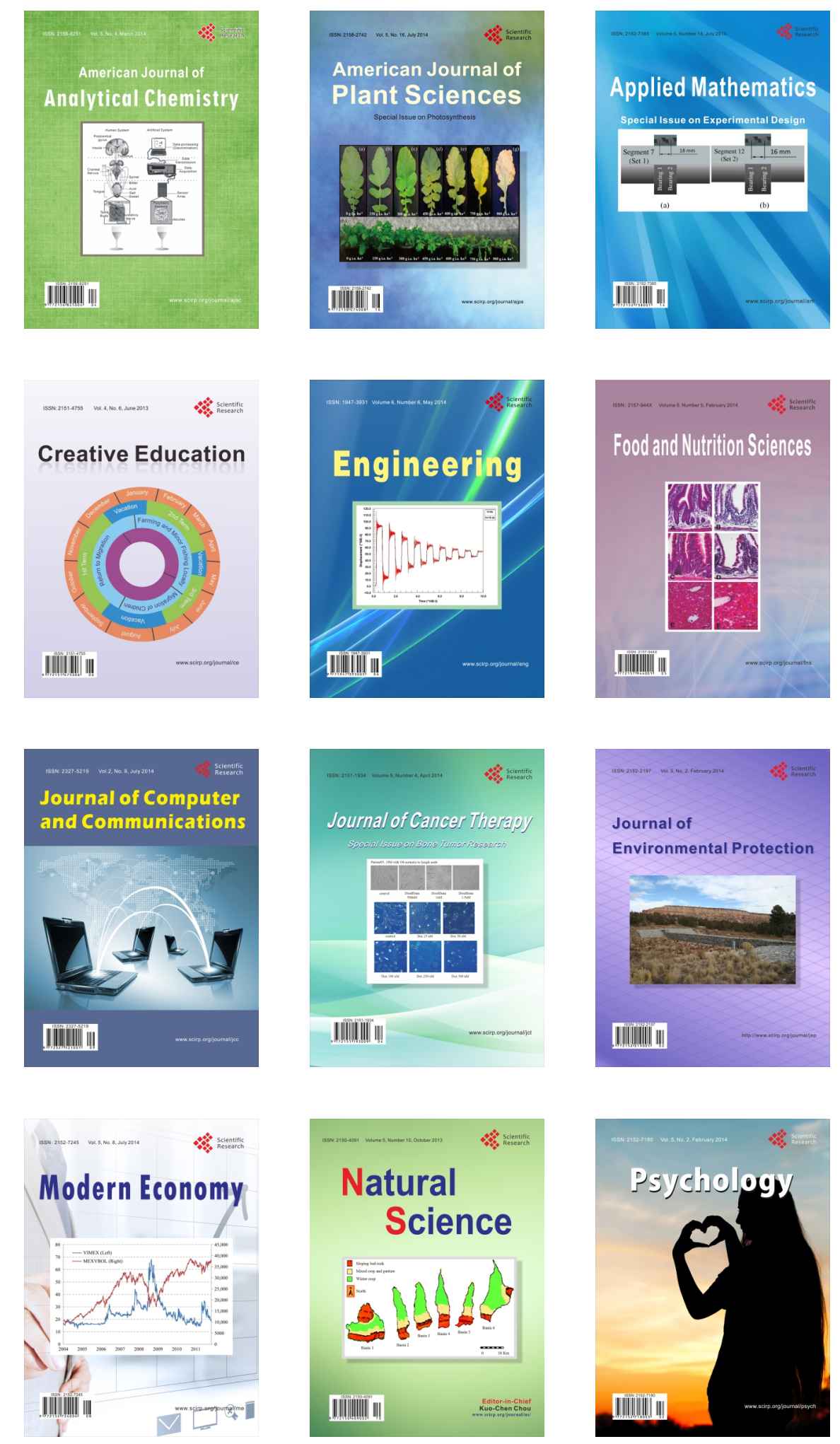